\title{
Synthesis and Properties of
}

\section{Hexakis(6-octyl-2-azulenyl)benzene as a Multielectron Redox System with Liquid Crystalline Behavior}

\author{
Shunji Ito, ${ }^{* \dagger}$ Mariko Ando, ${ }^{*}$ Akiko Nomura, ${ }^{*}$ Noboru Morita, ${ }^{+}$Chizuko Kabuto, ${ }^{+}$Hidetomo Mukai, ${ }^{\S}$ \\ Kazuchika Ohta, ${ }^{\S}$ Jun Kawakami, ${ }^{\dagger}$ Atsushi Yoshizawa, ${ }^{\dagger}$ and Akio Tajiri ${ }^{\dagger}$
}

Department of Materials Science and Technology, Faculty of Science and Technology, Hirosaki University, Hirosaki 036-8561, Japan, Department of Chemistry, Graduate School of Science, Tohoku University, Sendai 980-8578, Japan, and Department of Functional Polymer Science, Faculty of Textile Science and Technology, Shinshu University, Ueda 386-8567, Japan

\section{Supporting Information}

(1) Attempt for the preparation of 2-bromo- or 2-chloroazulene derivatives commenced with diethyl 6-bromo-2-aminoazulene-1,3-dicarboxylate

(2) ORTEP plots and structure feature of the X-ray analysis of compound 6

(3) Concentration dependence of the ${ }^{1} \mathrm{H}$ NMR spectrum of $\mathbf{1 b}$

(4) CV and DPV data of compounds $\mathbf{1 b}, \mathbf{2 b}$, and 19

S8

(5) X-ray diffraction powder patterns of compounds $\mathbf{1 b}, \mathbf{2 b}$, and 19

(6) Table of X-ray diffraction data of the mesophase of compounds $\mathbf{1 b}, \mathbf{2 b}$, and $\mathbf{1 9}$

(7) Proposed phase structure models for compounds $\mathbf{1 b}$ and $\mathbf{2 b}$

S23 
Attempt for the preparation of 2-bromo- or 2-chloroazulene derivatives commenced with diethyl 6-bromo-2-aminoazulene-1,3-dicarboxylate
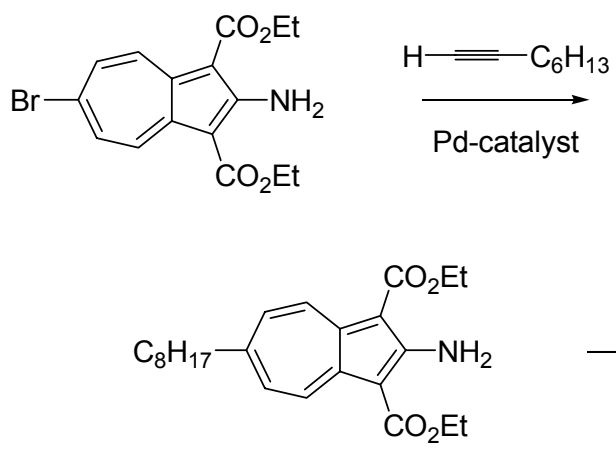
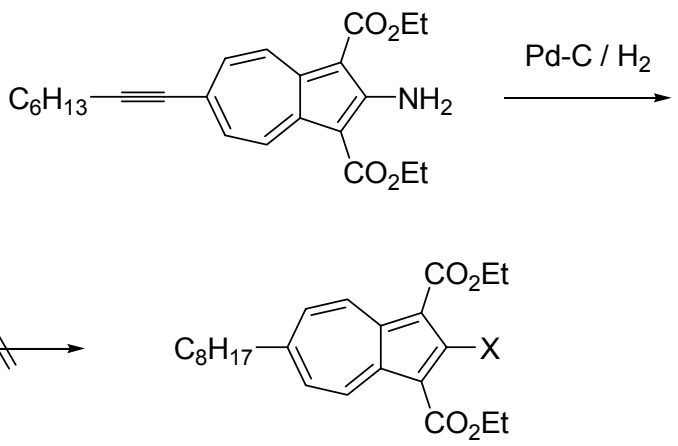

$\mathrm{X}=\mathrm{Br}$ or $\mathrm{Cl}$

Diethyl 2-Amino-6-(1-octynyl)azulene-1,3-dicarboxylate. To a degassed solution of diethyl 2-amino-6-bromoazulene-1,3-dicarboxylate (1.75 g, $4.78 \mathrm{mmol}), 1$-octyne (1.07 g, $9.71 \mathrm{mmol}), \mathrm{CuI}$ (98 $\mathrm{mg}, 0.51 \mathrm{mmol})$, triethylamine $(68 \mathrm{~mL})$ in dry toluene $(165 \mathrm{~mL})$ was added $\mathrm{Pd}\left(\mathrm{PPh}_{3}\right)_{4}(272 \mathrm{mg}, 0.24$ mmol). The resulting mixture was stirred at $70{ }^{\circ} \mathrm{C}$ for $2.5 \mathrm{~h}$ under an $\mathrm{Ar}$ atmosphere. The reaction mixture was washed successively with $10 \% \mathrm{NH}_{4} \mathrm{Cl}$ solution and water, dried over $\mathrm{MgSO}_{4}$, and concentrated under reduced pressure. The residue was purified by column chromatography on silica gel with $20 \%$ ethyl acetate/hexane to afford diethyl 2-amino-6-(1-octynyl)azulene-1,3-dicarboxylate (1.36 g, $72 \%$ ). orange prisms; mp 71.2-71.6 ${ }^{\circ} \mathrm{C}$ (hexane); $\mathrm{MS}(70 \mathrm{eV}) \mathrm{m} / z$ (relative intensity) $397\left(\mathrm{M}^{+}+2\right.$, 4.3\%), $396\left(\mathrm{M}^{+}+1,27\right), 395\left(\mathrm{M}^{+}, 100\right), 350(11), 349$ (15), 278 (14), 277 (42); IR (KBr disk) $v_{\max } 3492$ (m), 3349 (s), 2977 (m), 2957 (m), 2930 (m), 1688 (s), 1663 (s), 1599 (s), 1565 (m), 1534 (m), 1501 (m), 1478 (m), 1426 (s), 1385 (m), 1227 (m), 1167 (s), 1119 (s), 1107 (s), 1071 (m), 1030 (m), 855 (m), 793 (m), $529(\mathrm{~m}) \mathrm{cm}^{-1}$; UV-vis $\left(\mathrm{CH}_{2} \mathrm{Cl}_{2}\right) \lambda_{\max } 249$ (log $\varepsilon$ 4.54), $275 \mathrm{sh}$ (4.09), $330 \mathrm{sh}$ (4.75), 339 (4.84), 389 sh (4.05), $409 \mathrm{sh}$ (4.16), 429 (4.32), $463 \mathrm{sh}(3.46) \mathrm{nm} ;{ }^{1} \mathrm{H}$ NMR (400 MHz, $\left.\mathrm{CDCl}_{3}\right) \delta=8.96(\mathrm{~d}, 2 \mathrm{H}, J=$ $\left.11.5 \mathrm{~Hz}, \mathrm{H}_{4,8}\right), 7.79$ (br s, 2H, 2- $\left.\mathrm{NH}_{2}\right), 7.61\left(\mathrm{~d}, 2 \mathrm{H}, J=11.5 \mathrm{~Hz}, \mathrm{H}_{5,7}\right), 4.45(\mathrm{q}, 4 \mathrm{H}, J=7.1 \mathrm{~Hz}$, 1,3-CO $\left.\mathrm{CO}_{2} \mathrm{Et}\right), 2.46\left(\mathrm{t}, 2 \mathrm{H}, J=7.0 \mathrm{~Hz}, \mathrm{H}_{3^{\prime}}\right), 1.63\left(\mathrm{tt}, 2 \mathrm{H}, J=7.7,7.0 \mathrm{~Hz}, \mathrm{H}_{4^{\prime}}\right), 1.51-1.44\left(\mathrm{~m}, 2 \mathrm{H}, \mathrm{H}_{5^{\prime}}\right)$, $1.47\left(\mathrm{t}, 6 \mathrm{H}, J=7.1 \mathrm{~Hz}, 1,3-\mathrm{CO}_{2} \mathrm{Et}\right), 1.38-1.32\left(\mathrm{~m}, 4 \mathrm{H}, \mathrm{H}_{6^{\prime}, 7^{\prime}}\right), 0.92\left(\mathrm{~m}, 3 \mathrm{H}, J=7.0 \mathrm{~Hz}, \mathrm{H}_{8^{\prime}}\right) ;{ }^{13} \mathrm{C} \mathrm{NMR}$ 
$\left(100 \mathrm{MHz}, \mathrm{CDCl}_{3}\right) \delta=166.4\left(\mathrm{~s}, 1,3-\mathrm{CO}_{2} \mathrm{Et}\right), 162.3\left(\mathrm{C}_{2}\right), 145.4\left(\mathrm{C}_{3 \mathrm{a}, 8 \mathrm{a}}\right), 135.6\left(\mathrm{C}_{5,7}\right), 129.9\left(\mathrm{C}_{4,8}\right), 129.0$ $\left(\mathrm{C}_{6}\right), 100.3\left(\mathrm{C}_{1,3}\right), 93.6\left(\mathrm{C}_{2^{\prime}}\right), 84.2\left(\mathrm{C}_{1^{\prime}}\right), 59.9\left(\mathrm{t}, 1,3-\mathrm{CO}_{2} \mathrm{Et}\right), 31.3\left(\mathrm{C}_{6^{\prime}}\right), 28.6\left(\mathrm{C}_{5^{\prime}}\right), 28.6\left(\mathrm{C}_{4^{\prime}}\right), 22.6\left(\mathrm{C}_{7^{\prime}}\right)$, $19.7\left(\mathrm{C}_{3^{\prime}}\right), 14.6\left(\mathrm{q}, 1,3-\mathrm{CO}_{2} \mathrm{Et}\right), 14.1\left(\mathrm{C}_{8^{\prime}}\right)$. Anal. Calcd for $\mathrm{C}_{24} \mathrm{H}_{29} \mathrm{NO}_{4}: \mathrm{C}, 72.89 ; \mathrm{H}, 7.39 ; \mathrm{N}, 3.54$. Found: C, 72.67; H, 7.56; N, 3.53.

\section{Diethyl 2-Amino-6-octylazulene-1,3-dicarboxylate. A mixture of diethyl} 2-amino-6-(1-octynyl)azulene-1,3-dicarboxylate (967 mg, $2.45 \mathrm{mmol}), 10 \% \mathrm{Pd}-\mathrm{C}$ (95 mg) in EtOH (90 $\mathrm{mL}$ ) was stirred at room temperature for 2 days under an $\mathrm{H}_{2}$ atmosphere. After the Pd catalyst was removed by filtration, the solvent was removed under reduced pressure. The residue was purified by column chromatography on silica gel with $20 \%$ ethyl acetate/hexane to afford diethyl 2-amino-6-octylazulene-1,3-dicarboxylate $(907 \mathrm{mg}, 93 \%)$. yellow needles; $\mathrm{mp} \quad 78.1-78.5{ }^{\circ} \mathrm{C}$ (methanol); MS (70 eV) $m / z$ (relative intensity) $401\left(\mathrm{M}^{+}+2,4.4 \%\right), 400\left(\mathrm{M}^{+}+1,26\right), 399\left(\mathrm{M}^{+}, 100\right)$, 354 (10), 353 (11), 300 (10), 254 (14), 196 (13); IR (KBr disk) v $v_{\max } 3499$ (s), 3353 (s), 2977 (m), 2921 (s), $2870(\mathrm{~m}), 2853(\mathrm{~m}), 1680$ (s), 1657 (s), 1597 (s), 1578 (s), 1538 (m), 1509 (s), 1497 (m), 1478 (m), $1472(\mathrm{~m}), 1429(\mathrm{~s}), 1381(\mathrm{~m}), 1279(\mathrm{~m}), 1240(\mathrm{~m}), 1177(\mathrm{~s}), 1111(\mathrm{~s}), 1094(\mathrm{~m}), 1036(\mathrm{~m}), 949(\mathrm{~m}), 845$ (m), $791(\mathrm{~m}), 517(\mathrm{~m}) \mathrm{cm}^{-1}$; UV-vis $\left(\mathrm{CH}_{2} \mathrm{Cl}_{2}\right) \lambda_{\max } 247$ (log $\varepsilon$ 4.46), $265 \mathrm{sh}$ (4.34), 321 (4.71), 332 (4.82), 369 (3.91), 394 (3.92), 454 (3.50) nm; ${ }^{1} \mathrm{H}$ NMR (400 MHz, $\left.\mathrm{CDCl}_{3}\right) \delta=9.06(\mathrm{~d}, 2 \mathrm{H}, J=11.2 \mathrm{~Hz}$, $\mathrm{H}_{4,8}$ ), 7.68 (br s, 2H, 2-NH$), 7.45$ (d, 2H, $\left.J=11.2 \mathrm{~Hz}, \mathrm{H}_{5,7}\right), 4.45$ (q, 4H, J= 7.1 Hz, 1,3-CO $\left.{ }_{2} \mathrm{Et}\right), 2.77$ (t, $\left.2 \mathrm{H}, J=7.7 \mathrm{~Hz}, \mathrm{H}_{1^{\prime}}\right), 1.68\left(\mathrm{tt}, 2 \mathrm{H}, J=7.7,7.1 \mathrm{~Hz}, \mathrm{H}_{2^{\prime}}\right), 1.47\left(\mathrm{t}, 6 \mathrm{H}, J=7.1 \mathrm{~Hz}, 1,3-\mathrm{CO}_{2} \mathrm{Et}\right), 1.38-1.22$ $\left(\mathrm{m}, 10 \mathrm{H}, \mathrm{H}_{3^{\prime}, 4^{\prime}, 5^{\prime}, 6^{\prime}, 7^{\prime}}\right), 0.87\left(\mathrm{~m}, 3 \mathrm{H}, \mathrm{H}_{8^{\prime}}\right) ;{ }^{13} \mathrm{C} \mathrm{NMR}\left(100 \mathrm{MHz}, \mathrm{CDCl}_{3}\right) \delta=166.7\left(\mathrm{~s}, 1,3-\mathrm{CO}_{2} \mathrm{Et}\right), 161.9$ $\left(\mathrm{C}_{2}\right), 149.4\left(\mathrm{C}_{6}\right), 144.7\left(\mathrm{C}_{3 \mathrm{a}, 8 \mathrm{a}}\right), 133.8\left(\mathrm{C}_{5,7}\right), 131.2\left(\mathrm{C}_{4,8}\right), 99.4\left(\mathrm{C}_{1,3}\right), 59.7\left(\mathrm{q}, 1,3-\mathrm{CO}_{2} \mathrm{Et}\right), 41.1\left(\mathrm{C}_{1^{\prime}}\right)$, $32.6\left(\mathrm{C}_{2^{\prime}}\right), 31.8(\mathrm{t}), 29.4(\mathrm{t}), 29.2(\mathrm{t}, 2 \mathrm{C}), 22.6\left(\mathrm{C}_{7^{\prime}}\right), 14.7\left(\mathrm{q}, 1,3-\mathrm{CO}_{2} \mathrm{Et}\right), 14.1\left(\mathrm{q}, \mathrm{C}_{8^{\prime}}\right)$. Anal. Calcd for $\mathrm{C}_{24} \mathrm{H}_{33} \mathrm{NO}_{4}:$ C, 72.15; H, 8.33; N, 3.51. Found: C, 72.23; H, 8.28; N, 3.51 . 


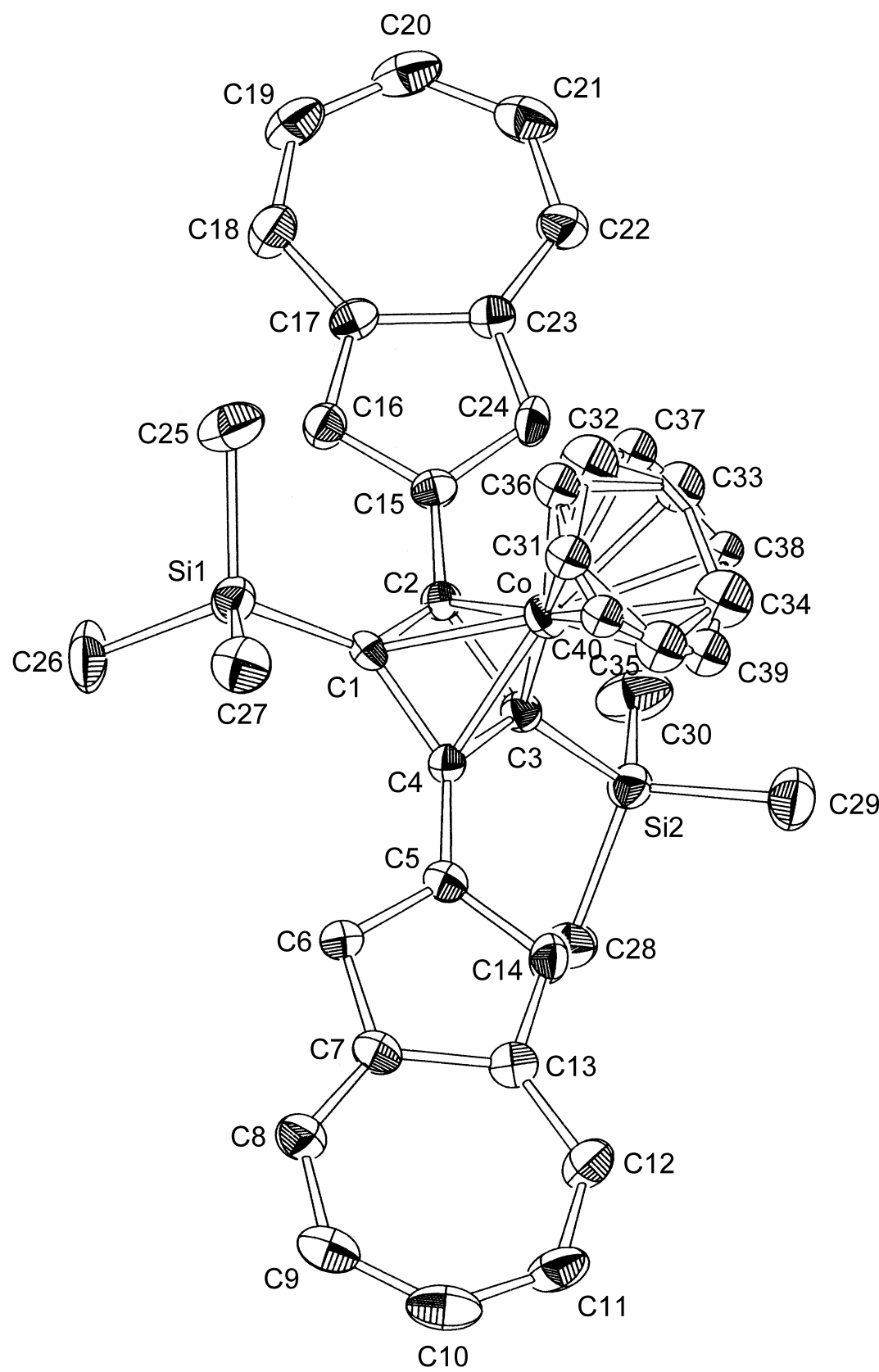

Figure S-1. ORTEP drawing of the molecule 6 in the crystalline state along with numbering scheme. Thermal ellipsoids are drawn at 30\% probability level. Selected bond lengthes $(\AA)$ : C(5) $-C(6)=1.40(1)$, $\mathrm{C}(5)-\mathrm{C}(14)=1.41(1), \mathrm{C}(6)-\mathrm{C}(7)=1.39(1), \mathrm{C}(7)-\mathrm{C}(8)=1.39(1), \mathrm{C}(7)-\mathrm{C}(13)=1.49(1), \mathrm{C}(8)-\mathrm{C}(9)=$ $1.38(1), \mathrm{C}(9)-\mathrm{C}(10)=1.39(2), \mathrm{C}(10)-\mathrm{C}(11)=1.38(2), \mathrm{C}(11)-\mathrm{C}(12)=1.36(1), \mathrm{C}(12)-\mathrm{C}(13)=1.38(1)$, $\mathrm{C}(13)-\mathrm{C}(14)=1.39(1), \mathrm{C}(15)-\mathrm{C}(66)=1.40(1), \mathrm{C}(15)-\mathrm{C}(24)=1.40(1), \mathrm{C}(16)-\mathrm{C}(17)=1.40(1)$, $\mathrm{C}(17)-\mathrm{C}(18)=1.38(1), \mathrm{C}(17)-\mathrm{C}(23)=1.50(1), \mathrm{C}(18)-\mathrm{C}(19)=1.38(1), \mathrm{C}(19)-\mathrm{C}(20)=1.38(2)$, $\mathrm{C}(20)-\mathrm{C}(21)=1.39(2), \mathrm{C}(21)-\mathrm{C}(22)=1.37(1), \mathrm{C}(22)-\mathrm{C}(23)=1.38(1), \mathrm{C}(23)-\mathrm{C}(24)=1.36(1)$. 


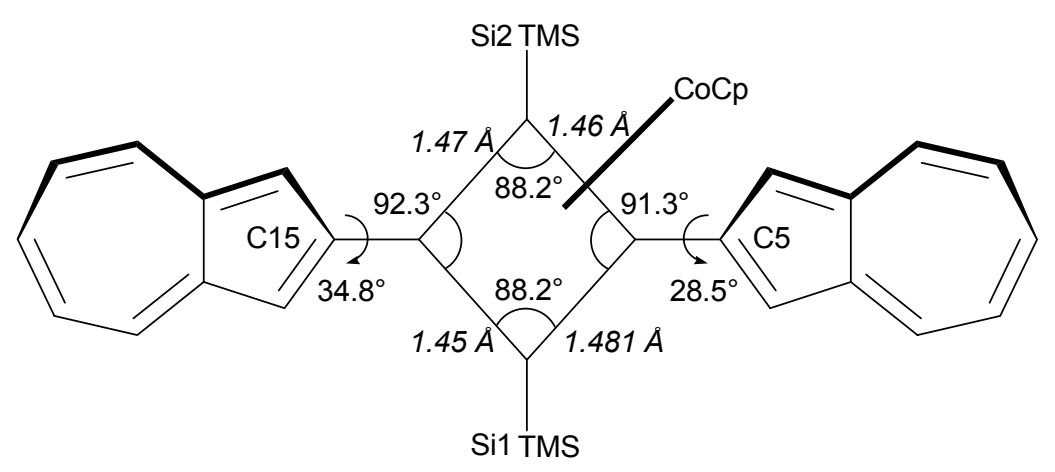

Figure S-2. Some key structural features in the cyclobutadiene ring of $\mathbf{6}$.

Data and diffraction parameters were obtained for a crystal with dimension $0.20 \times 0.20 \times 0.05$ mm using a Rigaku/MSC mercury CCD diffractometer with Mo-K $\alpha$ radiation $(\lambda=0.71070 \AA)$ at $-123{ }^{\circ} \mathrm{C}$. Crystal system: monoclinic. Space group: $P 2_{1} / n$ (\#14). Unit cell dimensions: $a=17.2663 \AA, b$ $=9.2015 \AA, c=21.2939 \AA, \beta=99.4733^{\circ}, \mathrm{V}=3336.9487 \AA^{3}, Z=4 . D_{\text {calcd }}=1.140 \mathrm{~g} \mathrm{~cm}^{-3} \cdot \mu(\mathrm{Mo}-\mathrm{K} \alpha)=$ $6.06 \mathrm{~cm}^{-1} . F(000)=1208.2 \theta$ range for data collection $=0.0-55.0^{\circ}$. Number of measured reflections $=$ 21979. Independent reflections $=7518\left(R_{\text {int }}=0.049\right)$. Final $R=0.055, R_{\mathrm{w}}=0.070$ for 2956 observed reflections $\left(I_{0}>4 \sigma\left(I_{0}\right)\right)$. Parameters $=394 . \mathrm{GOF}=1.54 . \Delta \rho_{\max }$ and $\Delta \rho_{\min }$ are 0.45 and $-0.28 \mathrm{e}^{-} \AA^{-3}$, respectively. Refinement method: full-matrix least squares. All calculations were performed using software package of Molecular Structure Corporation (Crystal Structure Analysis Package, Molecular Structure Corporation, 1985 \& 1999).

The cyclopentadienyl moiety of $\mathbf{6}$ is disordered in the crystal structure. The two azulene moieties have almost planar structures. The mean deviations from the least-squares plane of the two azulene moieties are $0.0050 \AA$ and $0.0089 \AA$, respectively. Each plane of the two azulene rings twisted in the same direction by $28.5^{\circ}$ and $34.8^{\circ}$, respectively, from the plane defined by the central cyclobutadiene ring. The cyclobutadiene ring is almost planar and forms a rhombus structure. Similar rhombus structure is observed in the crystal structure of trans- $\left(\eta^{5}\right.$-cyclopentadienyl)[bis $(1,3-$ diethoxycarbonyl-6-azulenyl)bis(trimethylsilyl)cyclobutadiene]cobalt. ${ }^{1}$

(1) Ito, S.; Inabe, H.; Okujima, T.; Morita, N.; Watanabe, M.; Harada, N.; Imafuku, K. J. Org. Chem. 2001, 66, 7090-7101. 


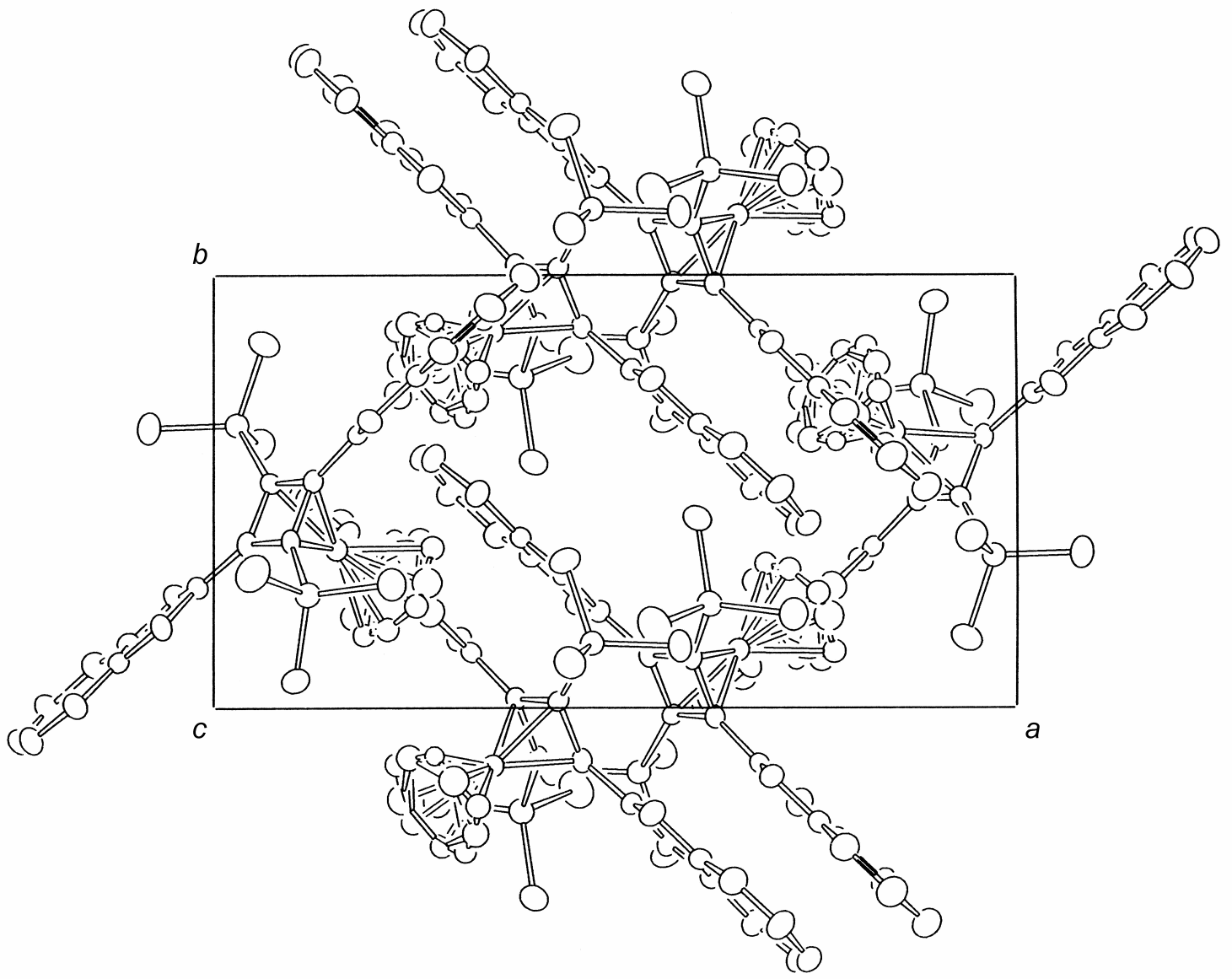

Figure S-3. Projection of the crystal structure of $\mathbf{6}$ along $c$-axis.

S6 


\section{Concentration dependence of the ${ }^{1} \mathrm{H}$ NMR spectrum of $1 \mathrm{~b}$}

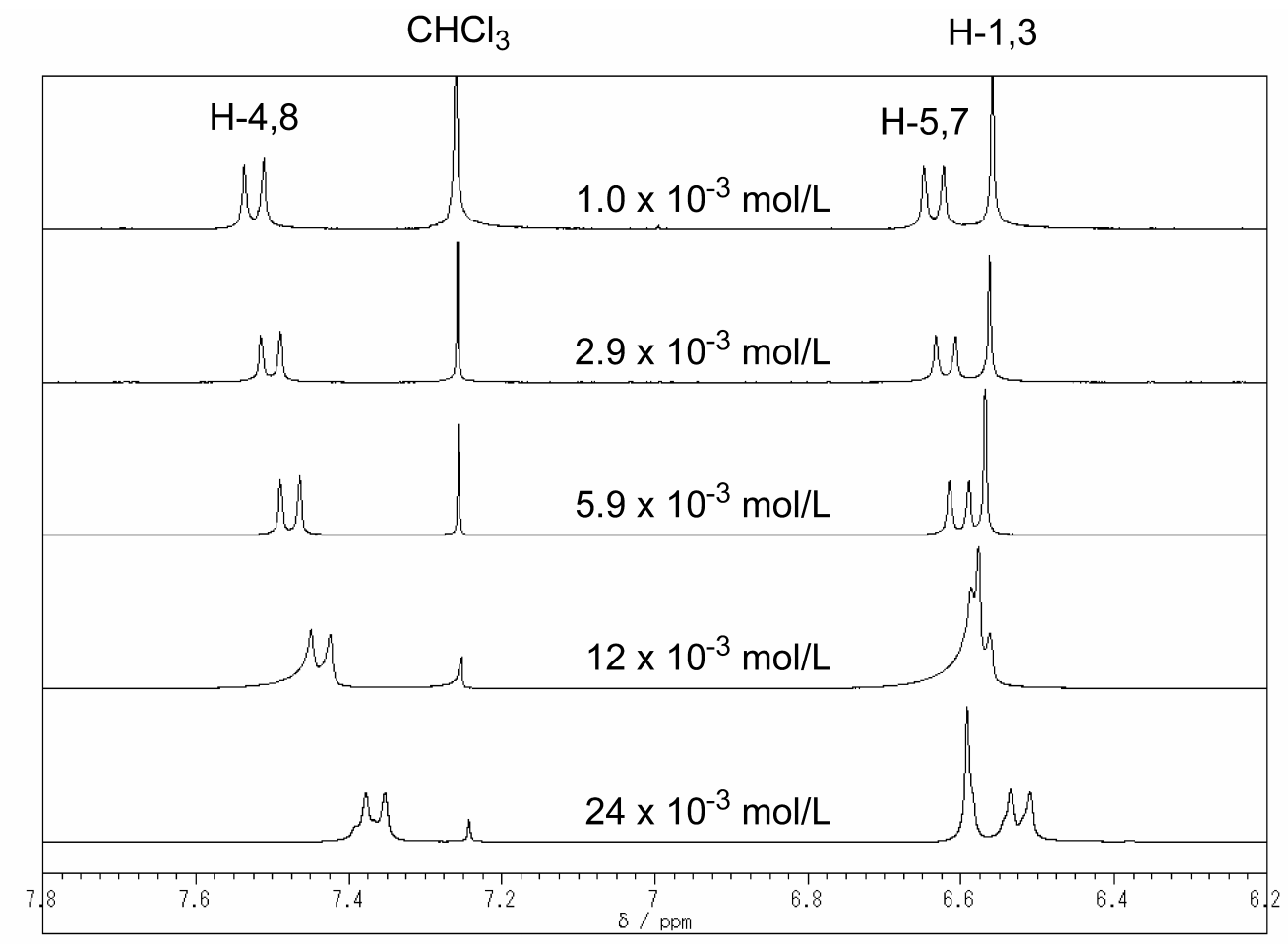

Figure S-4. Concentration dependence of the ${ }^{1} \mathrm{H}$ NMR spectrum of $\mathbf{1 b}$ in $\mathrm{CDCl}_{3}$. 


\section{Voltammograms}

(a)

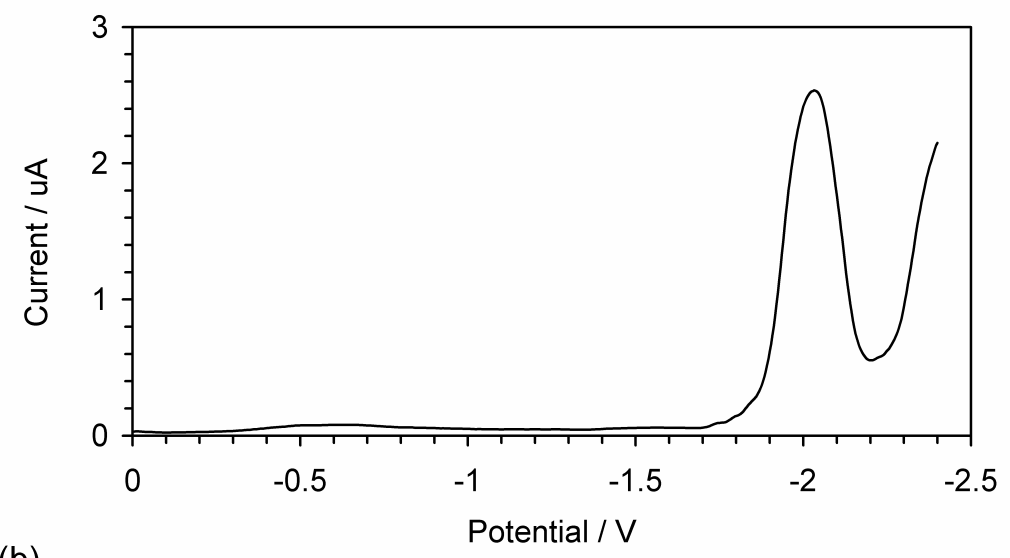

(b)

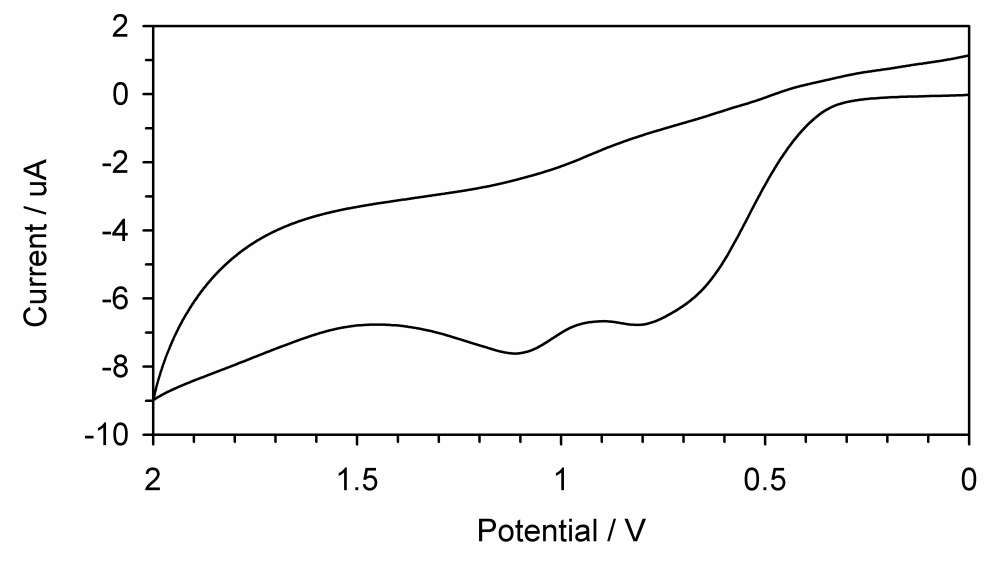

Figure S-5. (a) Reduction wave upon DPV and (b) oxidation wave upon CV of $\mathbf{1 b}(1 \mathrm{mM})$ in $o$-dichlorobenzene containing $n-\mathrm{Bu}_{4} \mathrm{NBF}_{4}(0.1 \mathrm{M})$ as a supporting electrolyte. 
(a)

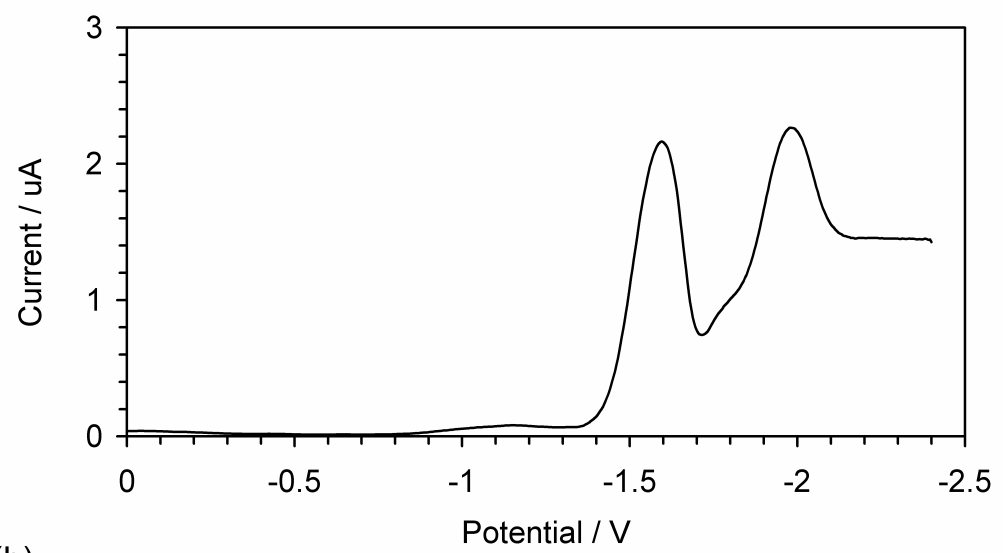

(b)

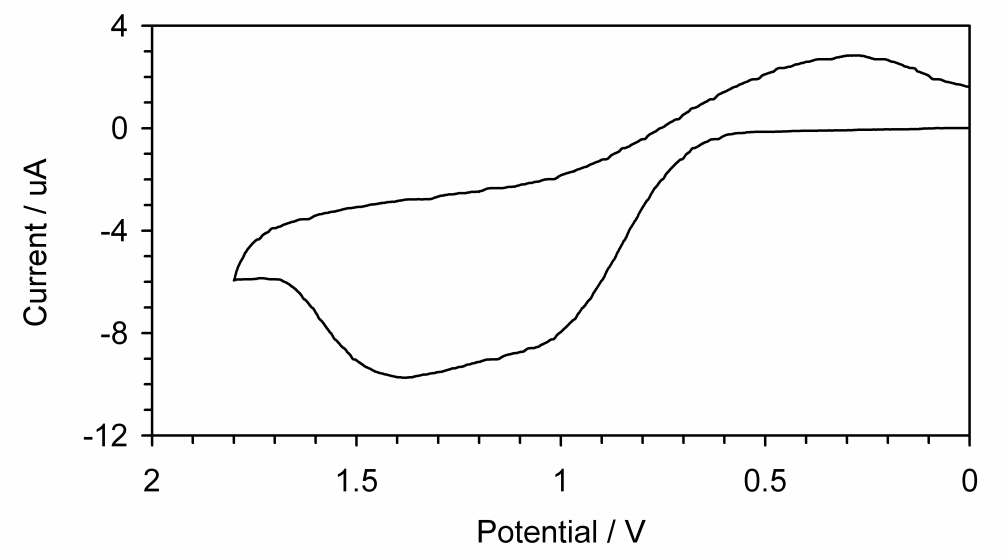

Figure S-6. (a) Reduction wave upon DPV and (b) oxidation wave upon CV of $2 \mathbf{b}(1 \mathrm{mM})$ in $o$-dichlorobenzene containing $n-\mathrm{Bu}_{4} \mathrm{NBF}_{4}(0.1 \mathrm{M})$ as a supporting electrolyte. 
(a)

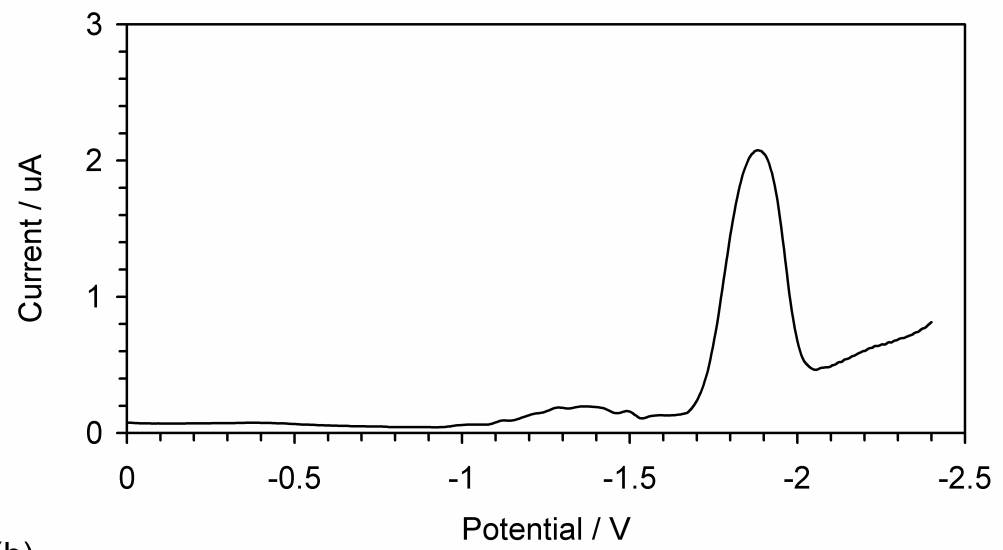

(b)

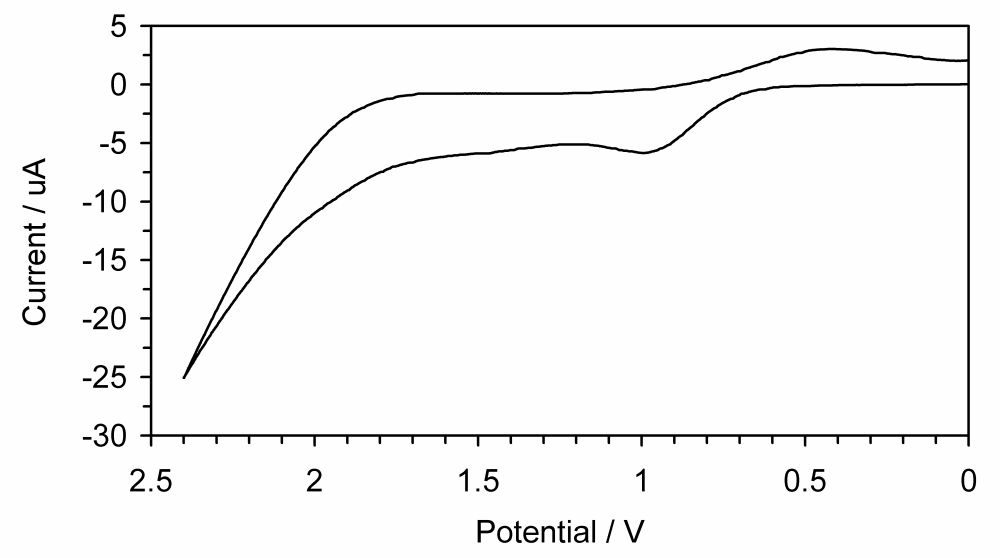

Figure S-7. (a) Reduction wave upon DPV and (b) oxidation wave upon CV of 19 (1 $\mathrm{mM})$ in $o$-dichlorobenzene containing $n-\mathrm{Bu}_{4} \mathrm{NBF}_{4}(0.1 \mathrm{M})$ as a supporting electrolyte. 
(a) at RT

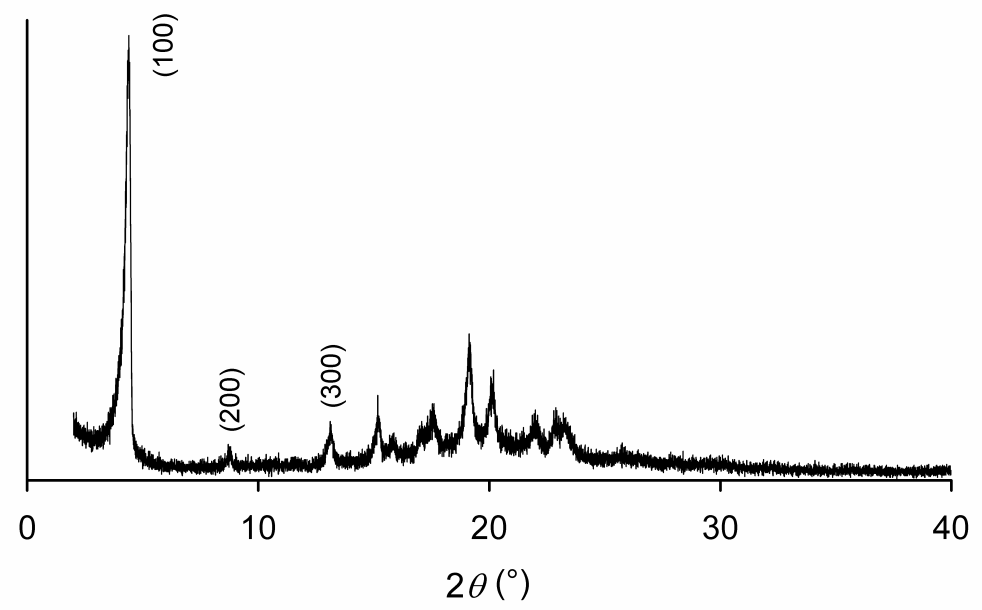

(b) at $99{ }^{\circ} \mathrm{C}$

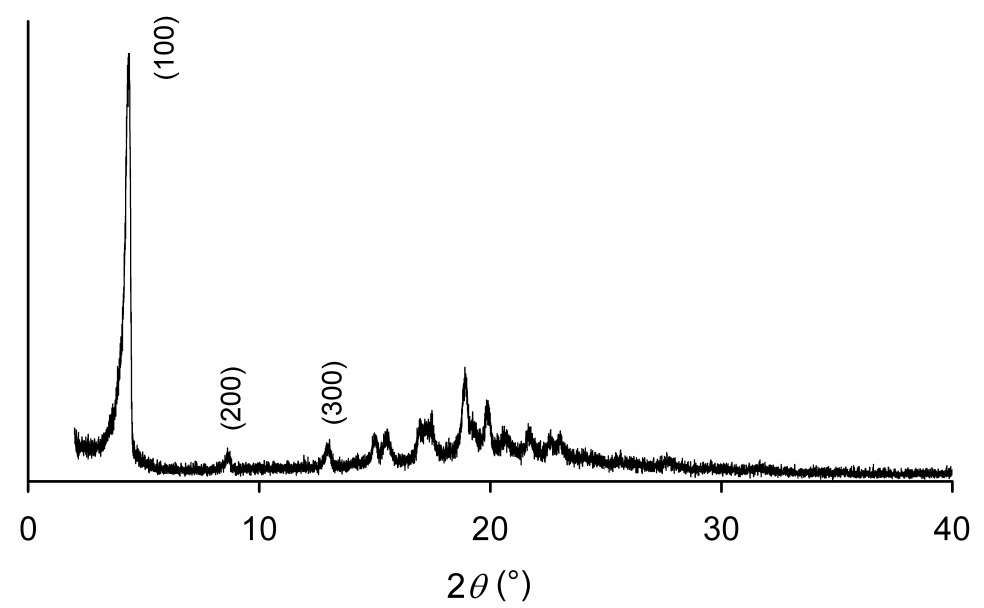

(c) at $114^{\circ} \mathrm{C}$

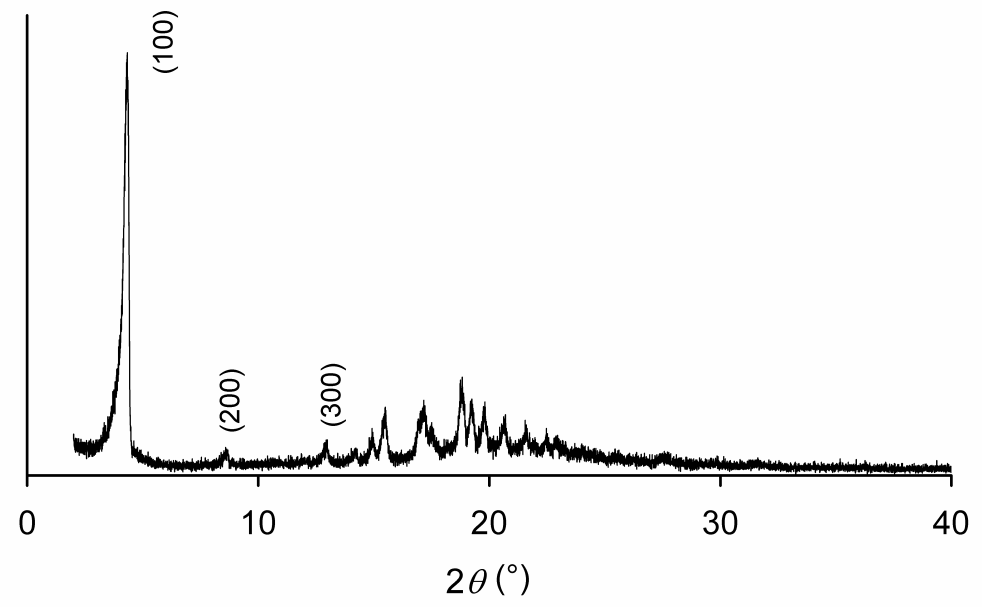

Figure S-8. X-ray diffraction powder patterns of $\mathbf{1 b}$ (a) at RT ( $\mathrm{K}_{1}$ phase), (b) at $99{ }^{\circ} \mathrm{C}\left(\mathrm{K}_{2}\right.$ phase), and (c) at $114{ }^{\circ} \mathrm{C}\left(\mathrm{K}_{3}\right.$ phase $)$. 
(a) at $123^{\circ} \mathrm{C}$

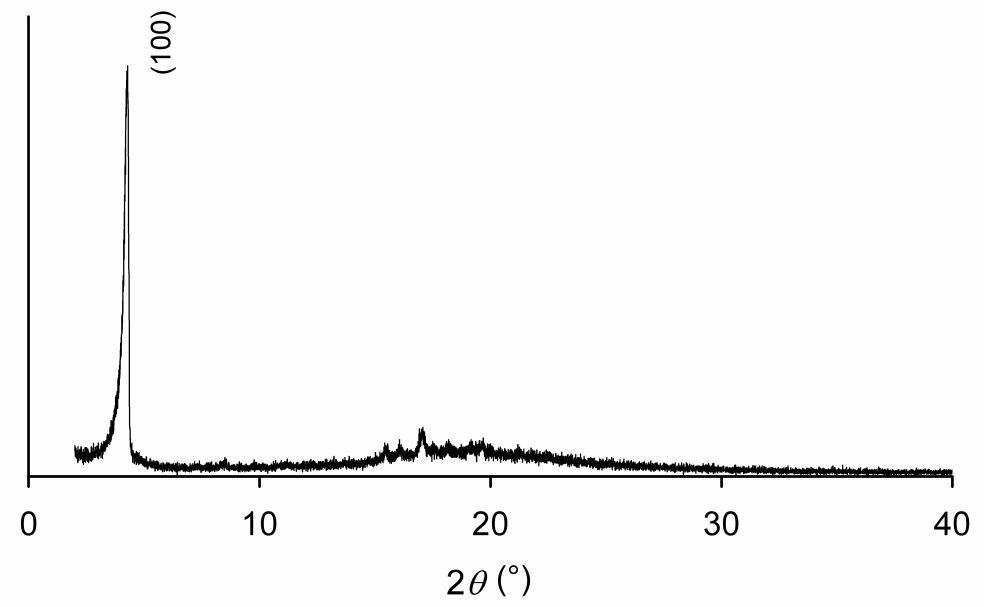

(b) at $123^{\circ} \mathrm{C}(\times 5)$

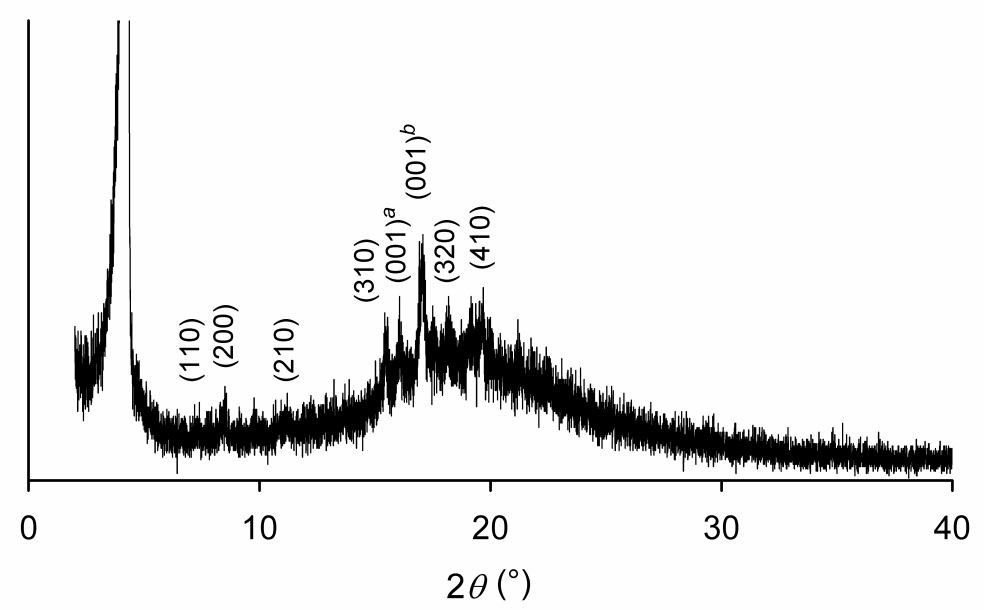

Figure S-9. (a) X-ray diffraction powder patterns of $\mathbf{1 b}$ at $\mathrm{Col}_{\mathrm{hol}}$ at $123{ }^{\circ} \mathrm{C}\left(\mathrm{Col}_{\mathrm{ho}}\right.$ mesophase) and (b) expansion. ${ }^{a} h_{2}=5.5 \AA .{ }^{b} h_{1}=5.2 \AA$. 
(a) at $133^{\circ} \mathrm{C}$

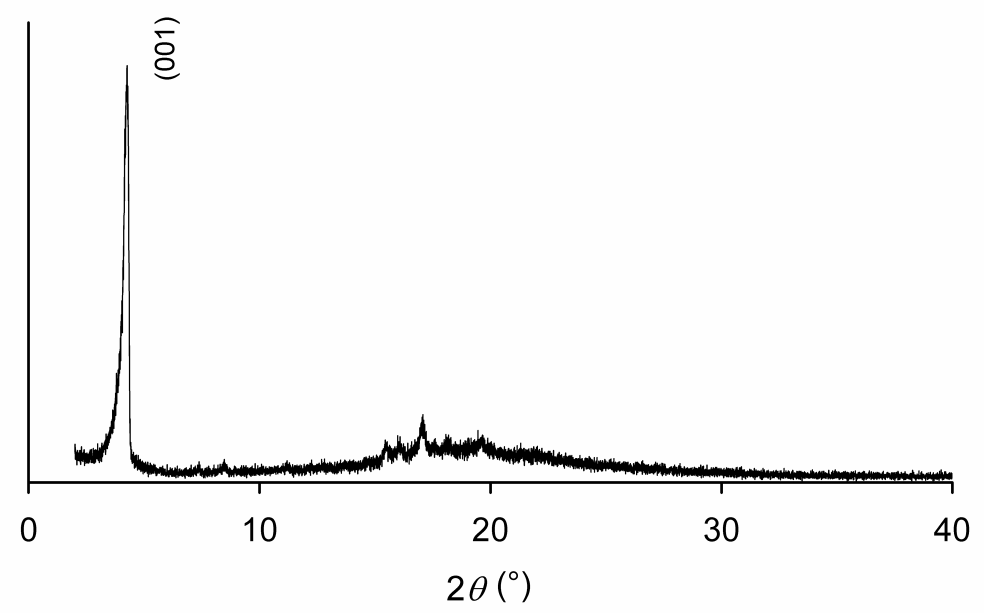

(b) at $133^{\circ} \mathrm{C}(\times 5)$

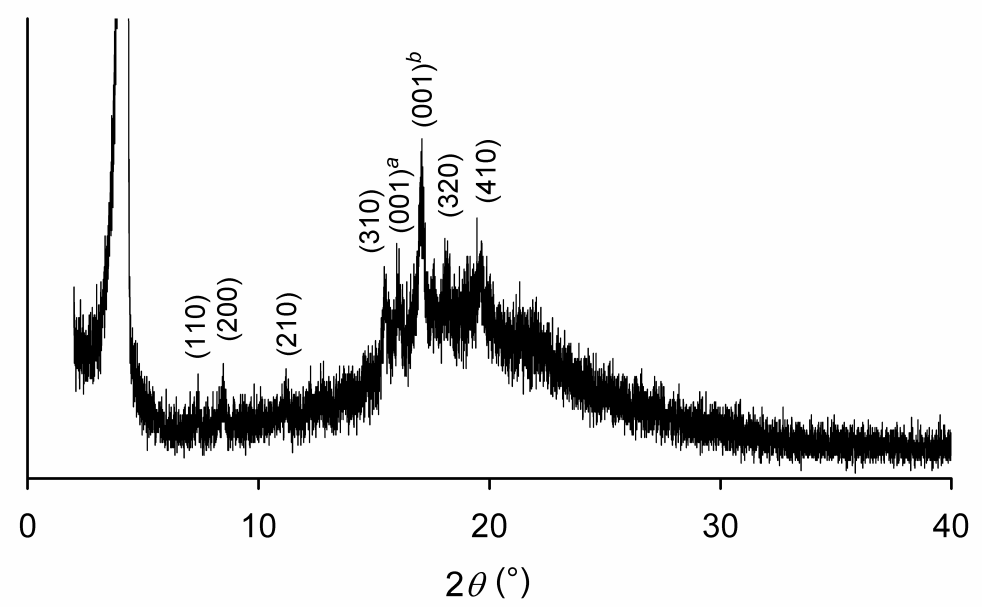

Figure S-10. (a) X-ray diffraction powder patterns of $\mathbf{1 b}$ at $\mathrm{Col}_{\mathrm{ho} 2}$ at $133{ }^{\circ} \mathrm{C}\left(\mathrm{Col}_{\mathrm{ho}}\right.$ mesophase) and (b) expansion. ${ }^{a} h_{2}=5.5 \AA .{ }^{b} h_{1}=5.2 \AA$. 
(a) at $141^{\circ} \mathrm{C}$

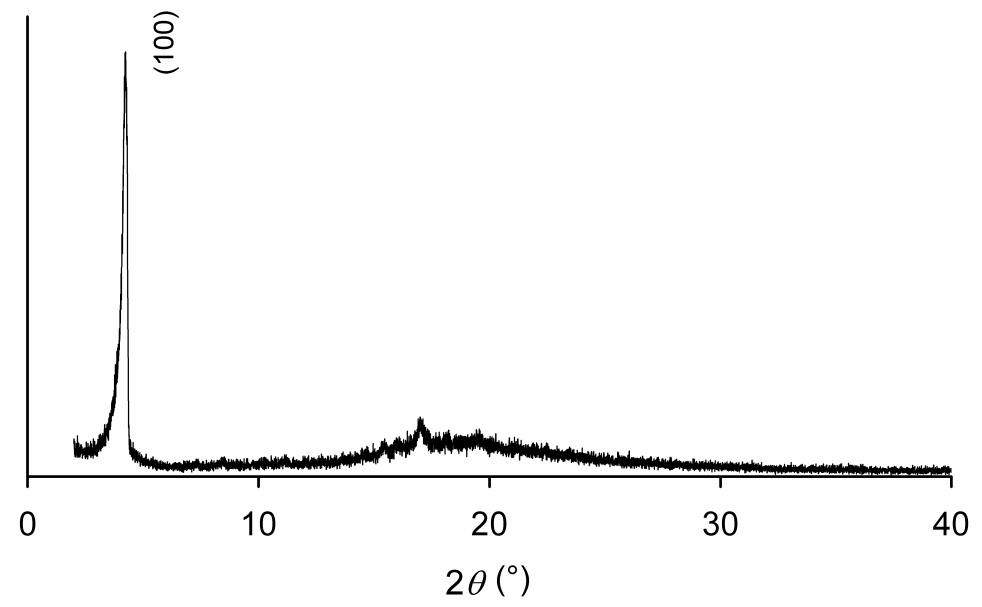

(b) at $141^{\circ} \mathrm{C}(\times 5)$

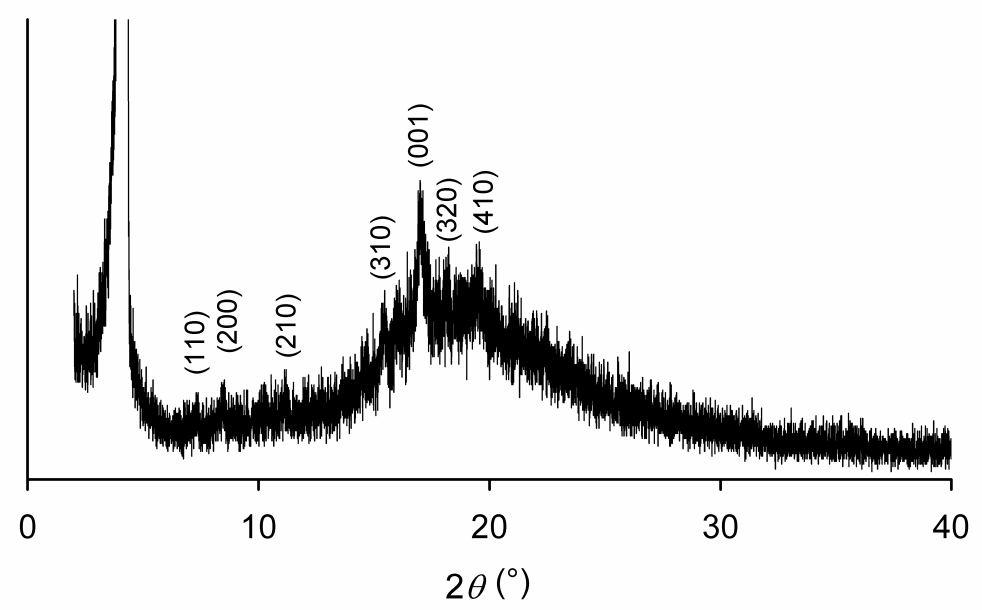

Figure S-11. (a) X-ray diffraction powder patterns of $\mathbf{1 b}$ at $\mathrm{Col}_{\mathrm{ho3}}$ at $141{ }^{\circ} \mathrm{C}\left(\mathrm{Col}_{\mathrm{ho}}\right.$ mesophase) and (b) expansion. 
(a) at $163^{\circ} \mathrm{C}$

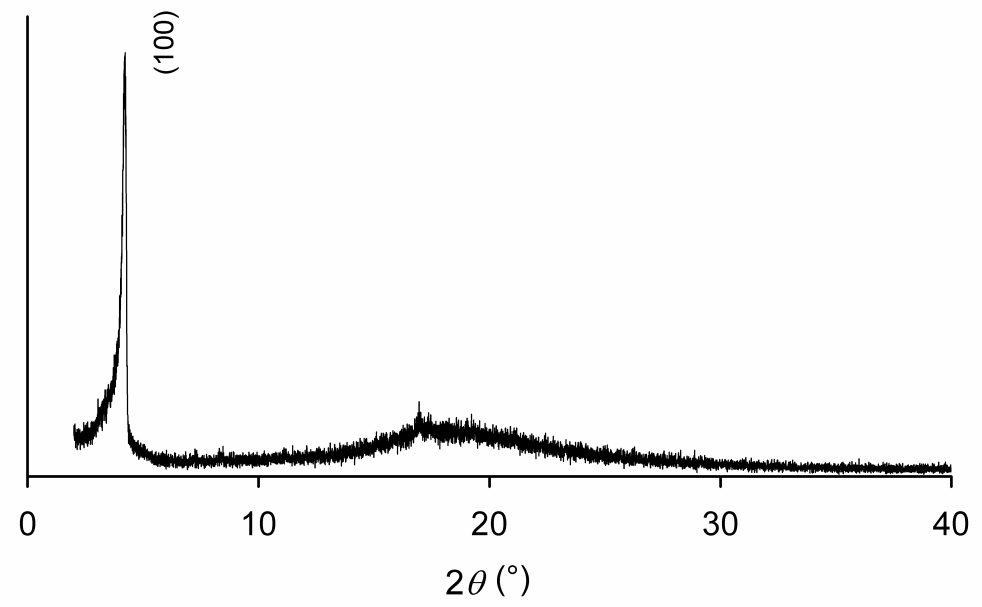

(b) at $163^{\circ} \mathrm{C}(\times 5)$

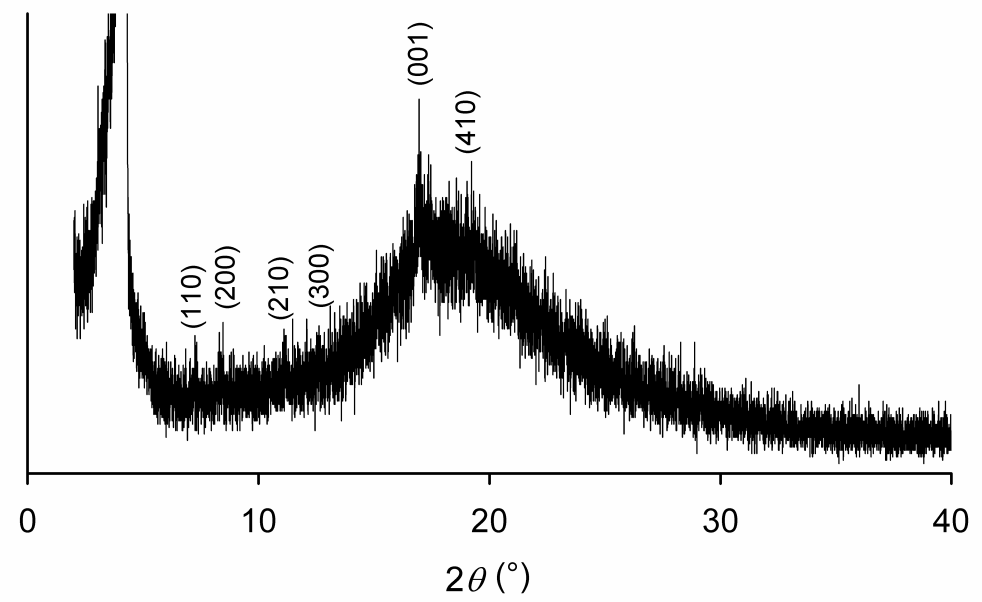

Figure S-12. (a) X-ray diffraction powder patterns of $\mathbf{1 b}$ at $\mathrm{Col}_{\mathrm{ho}}$ at $163{ }^{\circ} \mathrm{C}\left(\mathrm{Col}_{\mathrm{ho}}\right.$ mesophase) and (b) expansion. 


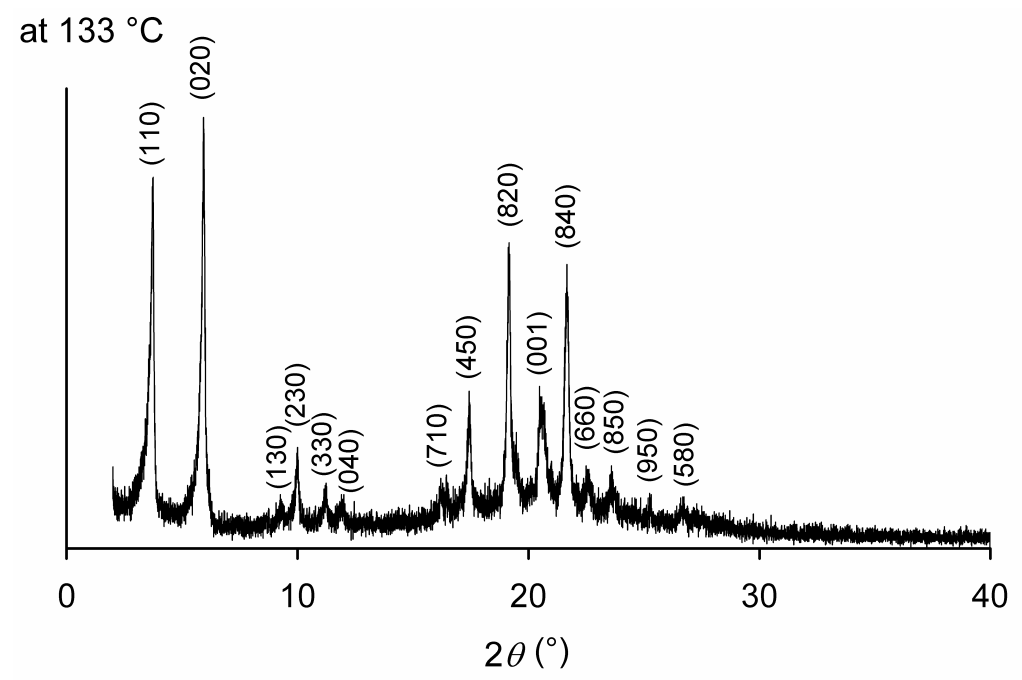

Figure S-13. (a) X-ray diffraction powder patterns of $\mathbf{2 b}$ at $133{ }^{\circ} \mathrm{C}\left[\mathrm{Col}_{\mathrm{ro}}\left(P 2_{1} / a\right)\right.$ mesophase]. 


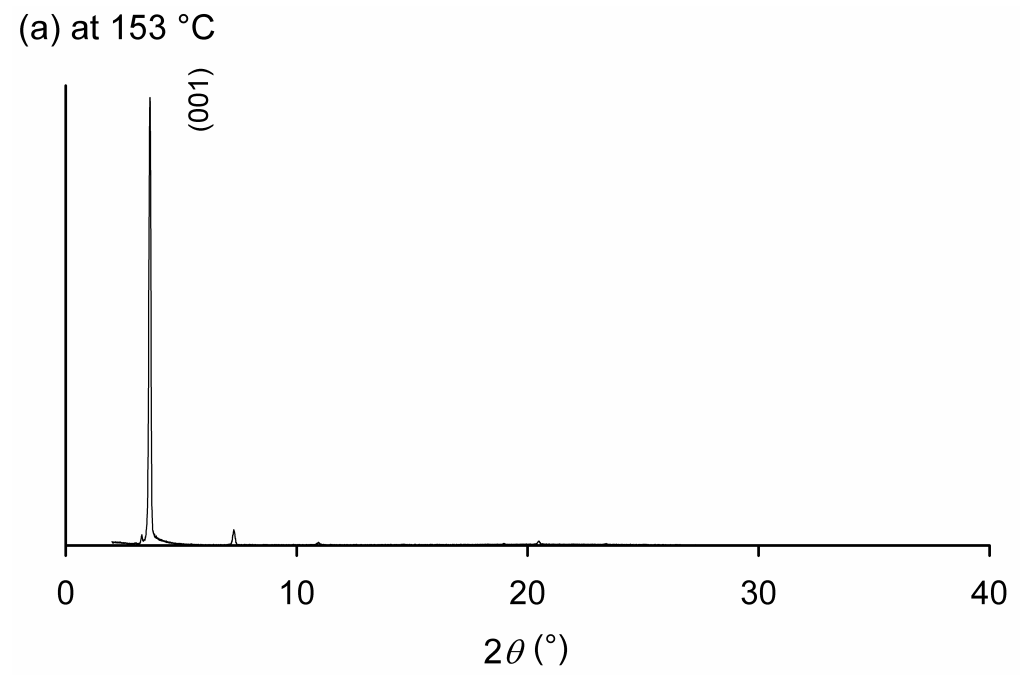

(b) at $153^{\circ} \mathrm{C}(\times 5)$

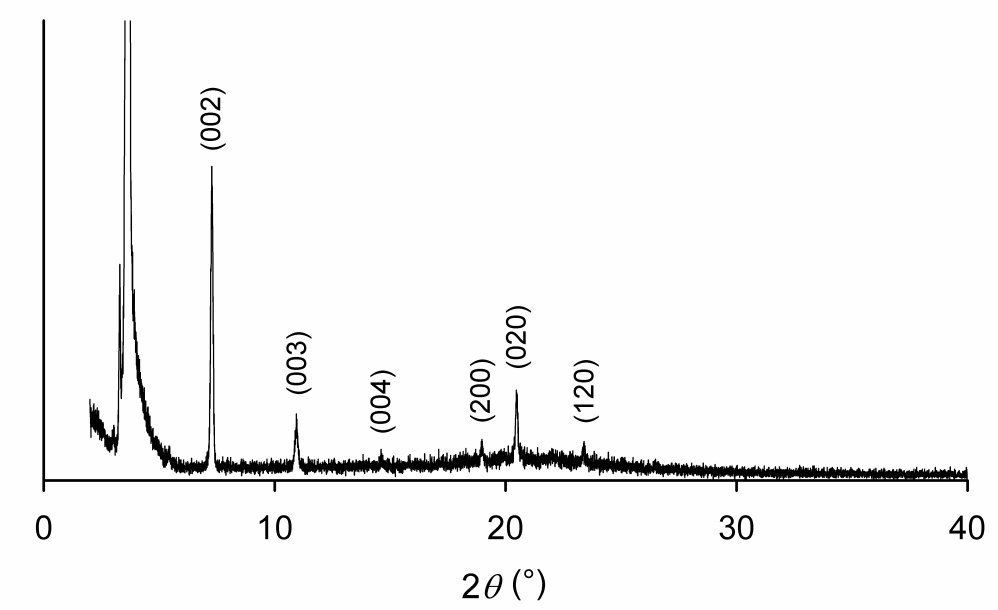

Figure S-14. (a) X-ray diffraction powder patterns of $\mathbf{2 b}$ at $153{ }^{\circ} \mathrm{C}\left(\mathrm{S}_{\mathrm{E}}\right.$ mesophase) and (b) expansion. 


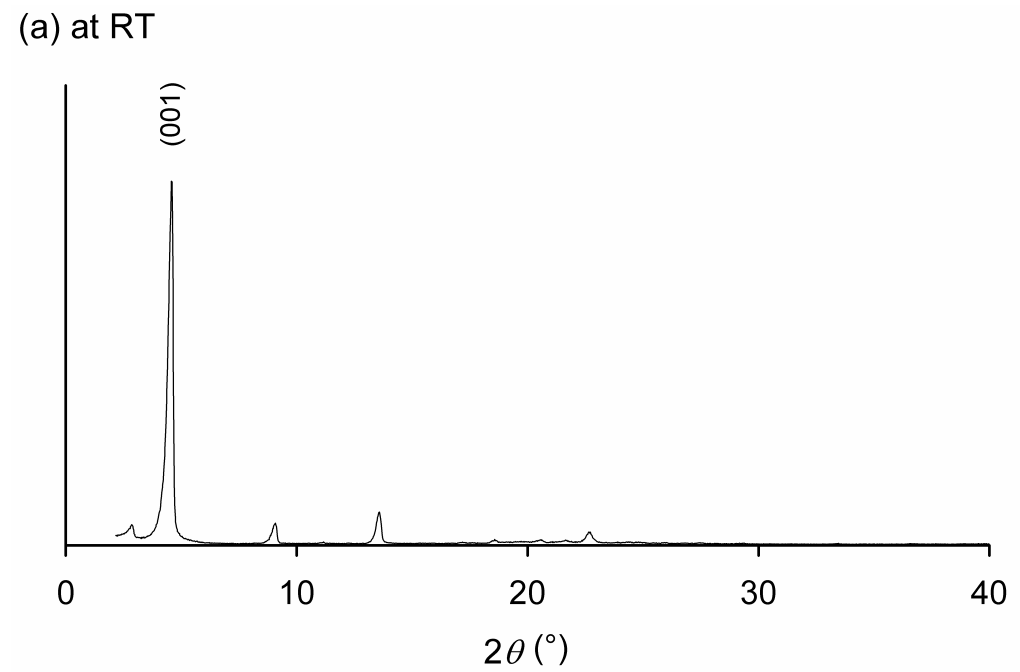

(b) at RT $(\times 10)$

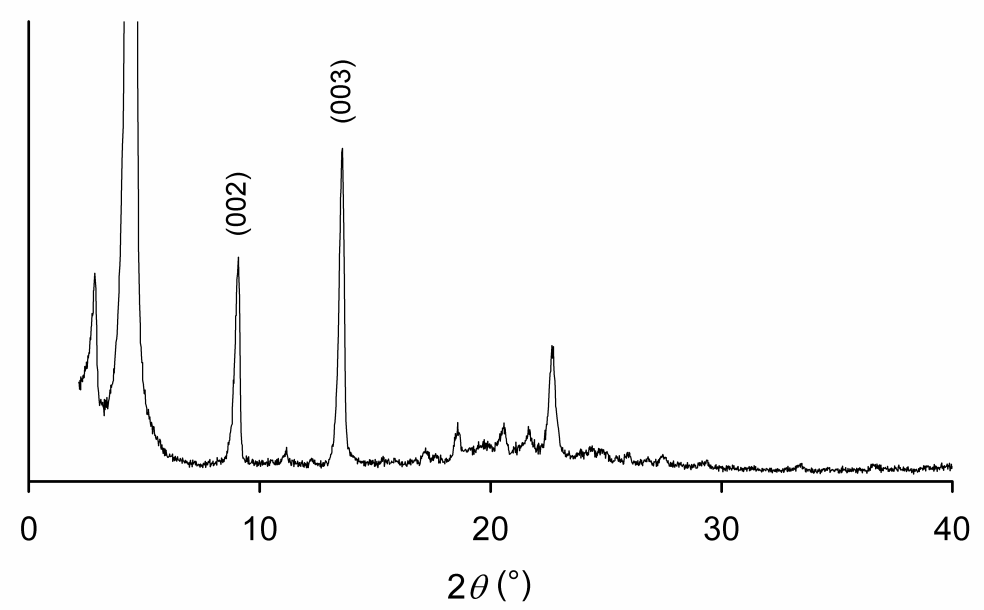

Figure S-15. (a) X-ray diffraction powder patterns of 19 at RT ( $\mathrm{K}_{1}$ phase) and (b) expansion. 


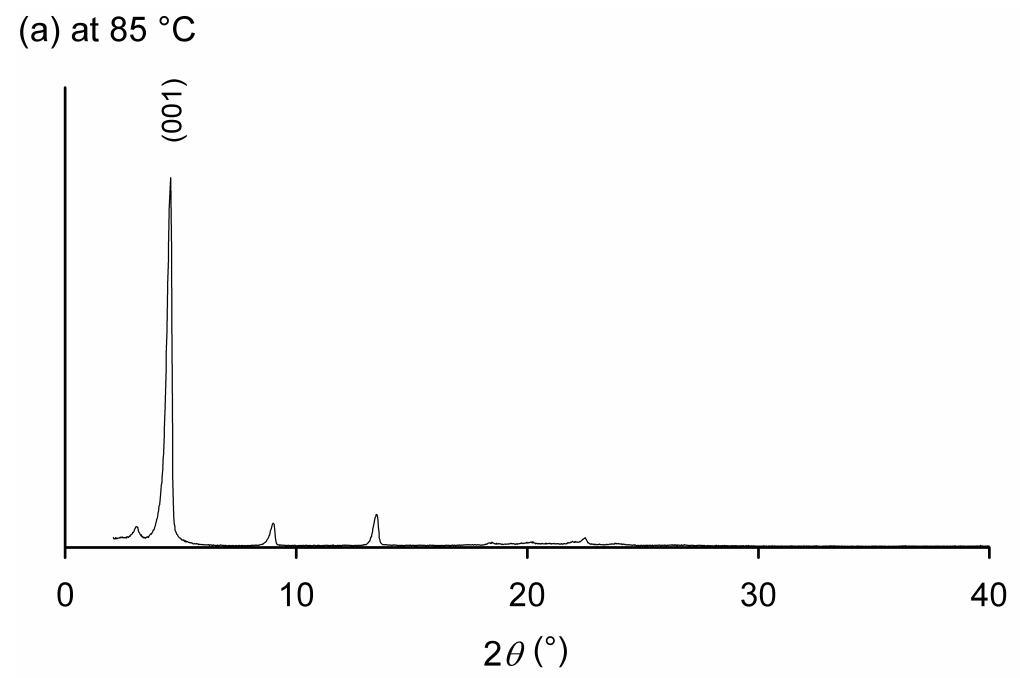

(b) at $85^{\circ} \mathrm{C}(\times 10)$

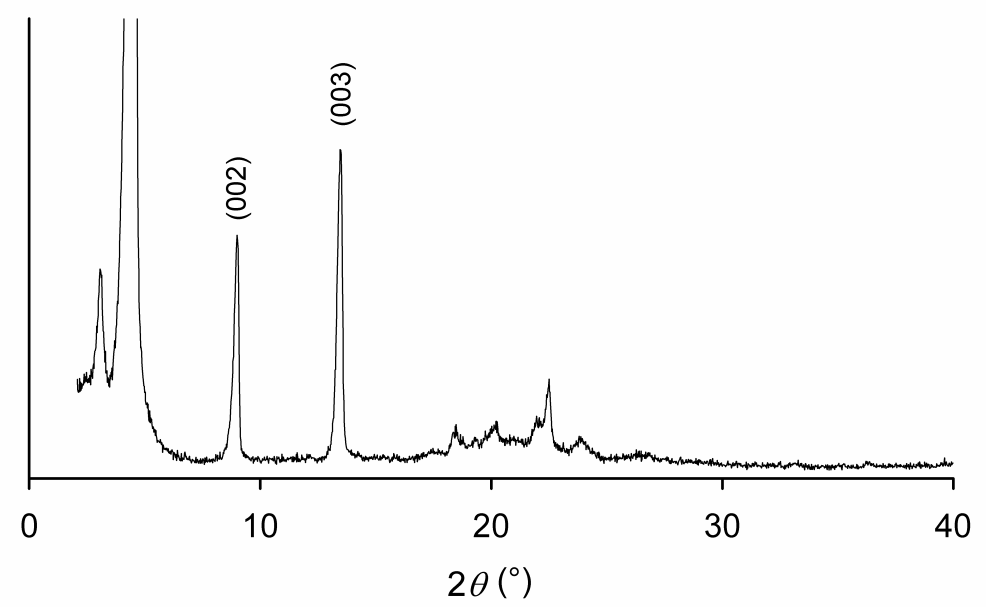

Figure S-16. (a) X-ray diffraction powder patterns of 19 at $85{ }^{\circ} \mathrm{C}\left(\mathrm{K}_{2}\right.$ phase $)$ and (b) expansion. 


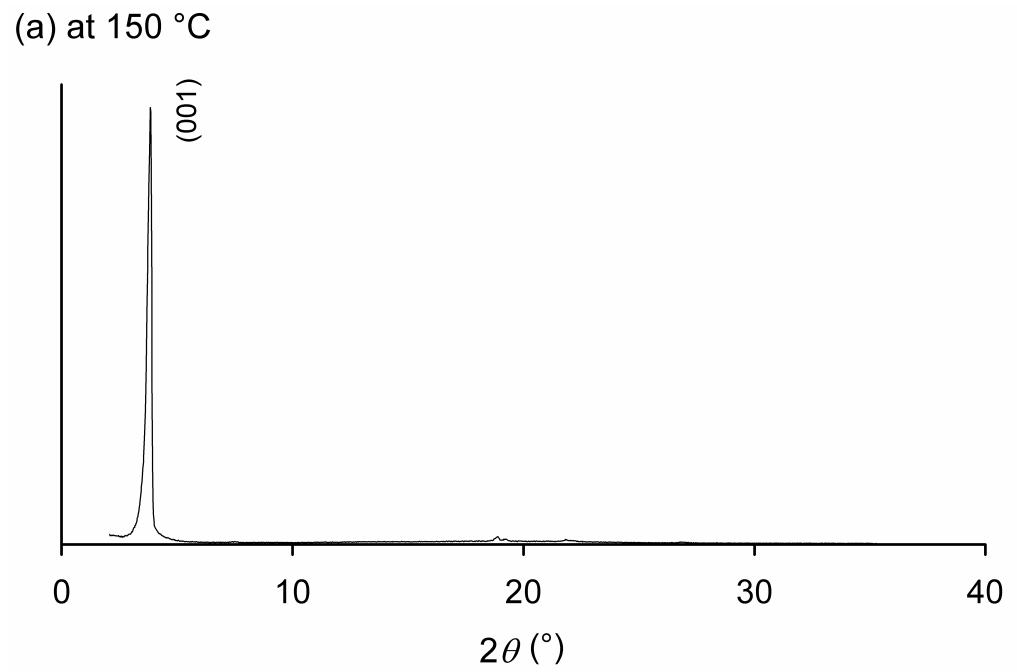

(b) at $150^{\circ} \mathrm{C}(\times 50)$

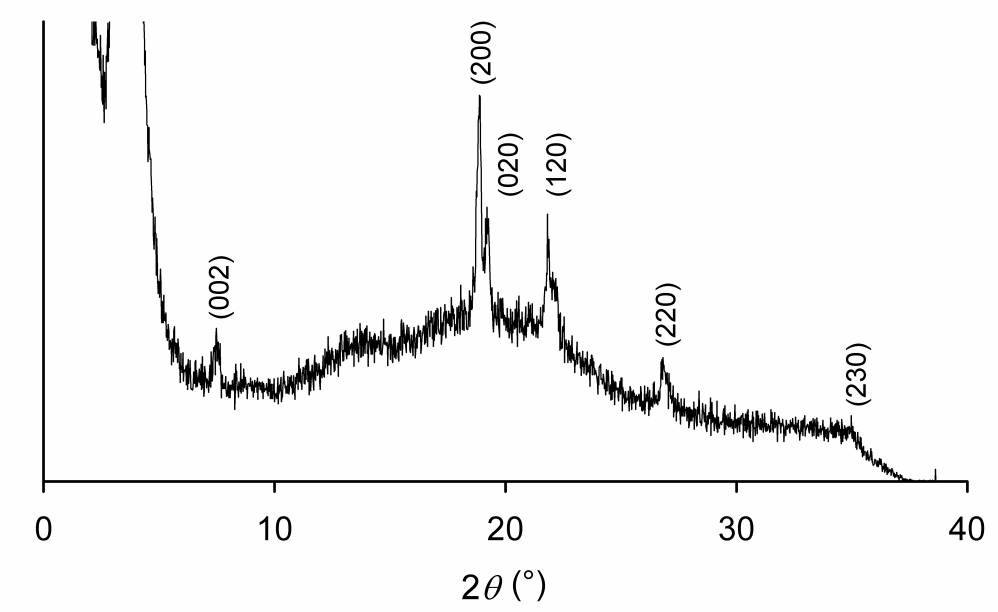

Figure S-17. (a) X-ray diffraction powder patterns of 19 at $150{ }^{\circ} \mathrm{C}\left(\mathrm{S}_{\mathrm{E}}\right.$ mesophase) and (b) expansion. 
Table S-1. X-Ray Diffraction Data of the Mesophase of Compound 1b

\begin{tabular}{|c|c|c|c|}
\hline \multirow[b]{2}{*}{ Lattice constants $/ \AA$} & \multicolumn{2}{|c|}{ Spacing/§ } & \multirow{2}{*}{$\begin{array}{l}\text { Miller indices } \\
(h k l)\end{array}$} \\
\hline & Observed & Calculated & \\
\hline $\mathrm{Col}_{\text {ho1 }}$ at $123^{\circ} \mathrm{C}$ & 20.7 & 20.7 & $(100)$ \\
\hline$a=23.9$ & 12.1 & 11.9 & $(110)$ \\
\hline$h=5.21$ & 10.4 & 10.3 & $(200)$ \\
\hline \multirow{6}{*}{$Z=1.0$ for $\rho^{a}=1.00$} & 7.98 & 7.82 & $(210)$ \\
\hline & 5.72 & 5.74 & $(310)$ \\
\hline & $5.52^{b}$ & & $(001)$ \\
\hline & $5.21^{b}$ & & $(001)$ \\
\hline & 4.89 & 4.75 & $(320)$ \\
\hline & 4.55 & 4.51 & $(410)$ \\
\hline $\mathrm{Col}_{\mathrm{ho} 2}$ at $133^{\circ} \mathrm{C}$ & 20.7 & 20.7 & $(100)$ \\
\hline$a=23.9$ & 12.0 & 11.9 & $(110)$ \\
\hline$h=5.20$ & 10.4 & 10.3 & $(200)$ \\
\hline \multirow[t]{6}{*}{$Z=1.0$ for $\rho^{a}=1.00$} & 7.90 & 7.82 & $(210)$ \\
\hline & 5.72 & 5.74 & $(310)$ \\
\hline & $5.52^{b}$ & & $(001)$ \\
\hline & $5.20^{b}$ & & $(001)$ \\
\hline & 4.88 & 4.75 & $(320)$ \\
\hline & 4.52 & 4.51 & $(410)$ \\
\hline $\mathrm{Col}_{\mathrm{ho} 3}$ at $141{ }^{\circ} \mathrm{C}$ & 20.8 & 20.8 & $(100)$ \\
\hline$a=24.0$ & 12.1 & 12.0 & $(110)$ \\
\hline$h=5.21$ & 10.4 & 10.4 & $(200)$ \\
\hline \multirow[t]{5}{*}{$Z=1.0$ for $\rho^{a}=1.00$} & 7.95 & 7.88 & $(210)$ \\
\hline & 5.75 & 5.78 & $(310)$ \\
\hline & 5.21 & & $(001)$ \\
\hline & 4.87 & 4.78 & $(320)$ \\
\hline & 4.55 & 4.55 & $(410)$ \\
\hline $\mathrm{Col}_{\mathrm{ho} 4}$ at $163^{\circ} \mathrm{C}$ & 21.0 & 21.0 & $(100)$ \\
\hline$a=24.2$ & 12.1 & 12.1 & $(110)$ \\
\hline$h=5.23$ & 10.5 & 10.5 & $(200)$ \\
\hline \multirow{4}{*}{$Z=1.0$ for $\rho^{a}=1.00$} & 7.92 & 7.94 & $(210)$ \\
\hline & 7.01 & 7.00 & $(300)$ \\
\hline & 5.23 & & $(001)$ \\
\hline & 4.66 & 4.58 & $(410)$ \\
\hline
\end{tabular}

${ }^{a}$ Assumed density (grams per cubic centimeter).

${ }^{b}$ These reflections could not be indexed into any spacing of 2D hexagonal and 3D hexagonal lattices. Thus, these reflections indicate that there are two different stacking distances in the mesophase. For the presumed stacking model, see Figure S-18. 
Table S-2. X-Ray Diffraction Data of the Mesophase of Compounds $\mathbf{2 b}$ and $\mathbf{1 9}$

\begin{tabular}{|c|c|c|c|}
\hline \multirow[b]{2}{*}{ Lattice constants $/ \AA$} & \multicolumn{2}{|c|}{ Spacing $/ \AA$} & \multirow{2}{*}{$\begin{array}{l}\text { Miller indices } \\
\qquad(h k l)\end{array}$} \\
\hline & Observed & Calculated & \\
\hline compound $\mathbf{2 b}$ & 23.7 & 23.7 & $(110)$ \\
\hline$\left(\mathrm{Col}_{\mathrm{ro}}\left(P 2_{1} / a\right)\right.$ at $\left.133{ }^{\circ} \mathrm{C}\right)$ & 14.9 & 14.9 & $(020)$ \\
\hline$a=38.9$ & 9.55 & 9.62 & $(130)$ \\
\hline$b=29.8$ & 8.83 & 8.85 & $(230)$ \\
\hline$h=4.32$ & 7.87 & 7.89 & $(330)$ \\
\hline \multirow{10}{*}{$Z=6.0$ for $\rho^{a}=1.00$} & 7.41 & 7.45 & $(040)$ \\
\hline & 5.47 & 5.46 & $(710)$ \\
\hline & 5.08 & 5.08 & $(450)$ \\
\hline & 4.63 & 4.62 & $(820)$ \\
\hline & 4.32 & & $(001)$ \\
\hline & 4.10 & 4.07 & $(840)$ \\
\hline & 3.94 & 3.94 & $(660)$ \\
\hline & 3.77 & 3.77 & $(850)$ \\
\hline & 3.52 & 3.50 & $(950)$ \\
\hline & 3.34 & 3.36 & $(580)$ \\
\hline compound $\mathbf{2 b}$ & 24.2 & 24.2 & $(001)$ \\
\hline$\left(\mathrm{S}_{\mathrm{E}}\right.$ at $\left.153^{\circ} \mathrm{C}\right)$ & 12.1 & 12.1 & $(002)$ \\
\hline$a=9.35$ & 8.08 & 8.07 & $(003)$ \\
\hline$b=8.67$ & 6.06 & 6.05 & $(004)$ \\
\hline$c=24.2$ & 4.67 & 4.67 & $(200)$ \\
\hline \multirow[t]{2}{*}{$Z=2.1$ for $\rho^{a}=0.90$} & 4.34 & 4.34 & $(020)$ \\
\hline & 3.80 & 3.93 & $(120)$ \\
\hline compound 19 & 23.0 & 23.0 & $(001)$ \\
\hline$\left(\mathrm{S}_{\mathrm{E}}\right.$ at $\left.150^{\circ} \mathrm{C}\right)$ & 11.8 & 11.5 & $(002)$ \\
\hline$a=9.33$ & 4.70 & 4.70 & $(200)$ \\
\hline$b=9.22$ & 4.62 & 4.62 & $(020)$ \\
\hline$c=23.0$ & 4.07 & 4.07 & $(120)$ \\
\hline \multirow{2}{*}{$Z=4.0$ for $\rho^{a}=1.05$} & 3.32 & 3.32 & $(220)$ \\
\hline & 2.56 & 2.56 & $(230)$ \\
\hline
\end{tabular}

${ }^{a}$ Assumed density (grams per cubic centimeter). 


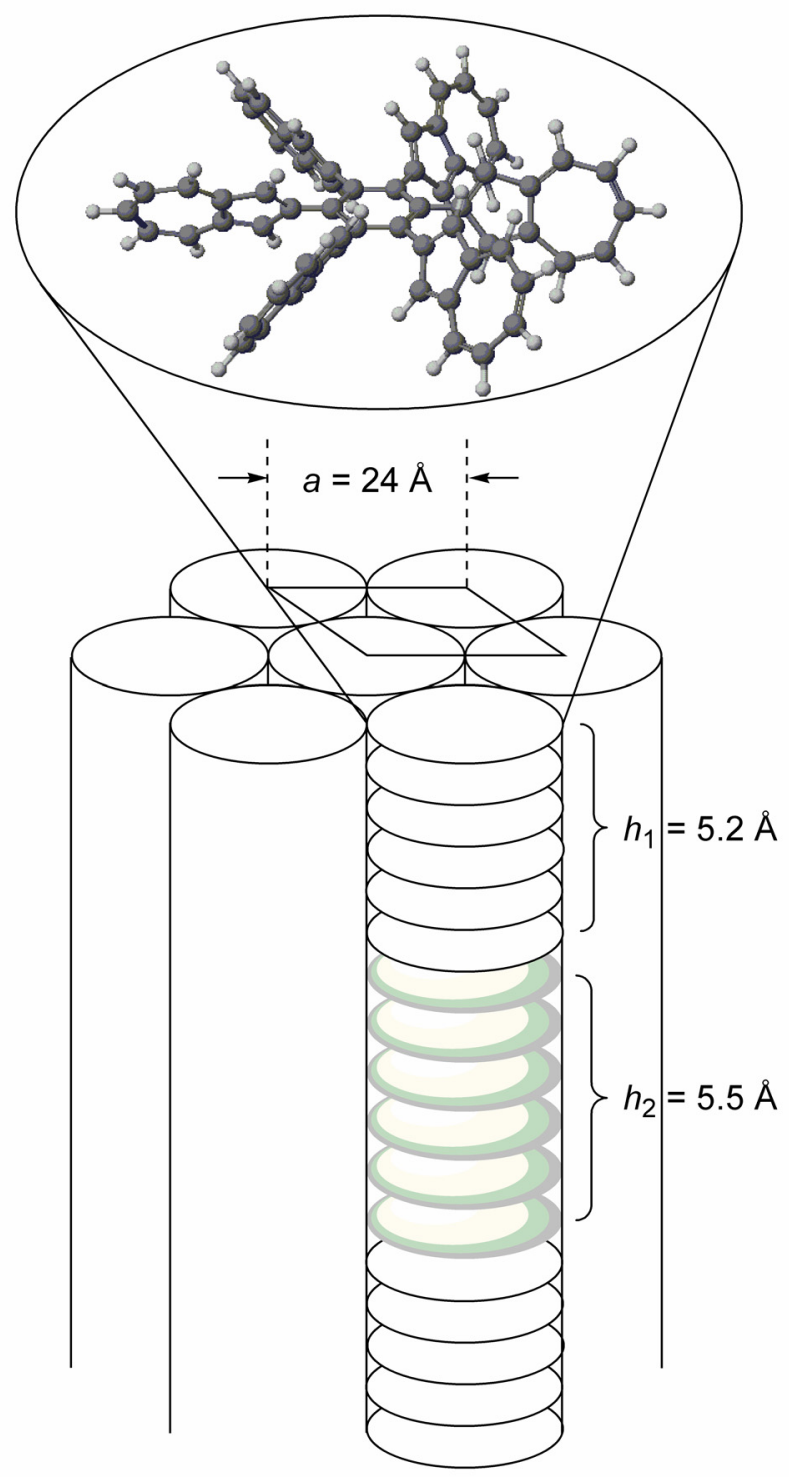

Figure S-18. A proposed phase structure model of the $\mathrm{Col}_{\text {ho }}$ mesophase of compound $\mathbf{1 b}$ with optimized molecular structure without 6-octyl chains calculated by MM2 method. ${ }^{2}$ The atom distances between 4and 8-positions of each azulene ring are $5.39 \AA$.

(2) MM2 calculation was performed by Tektronix CAChe WorkSystem. 


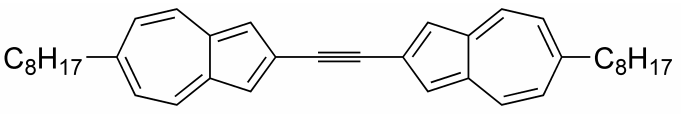

$2 b$
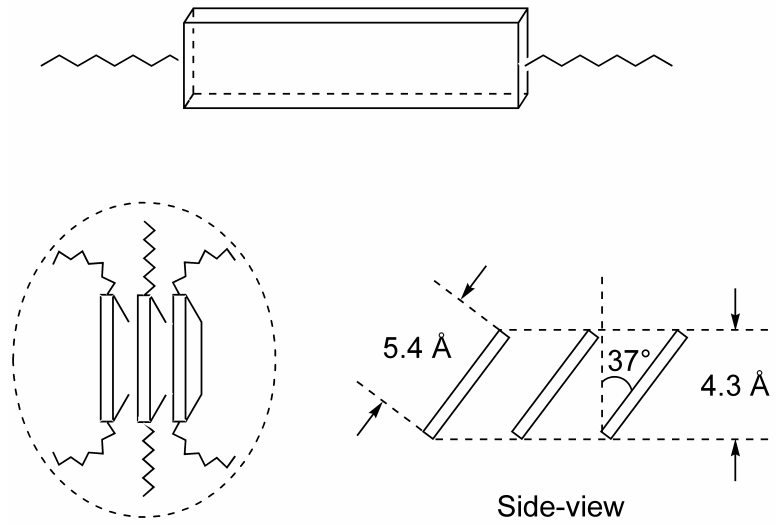

Top-view

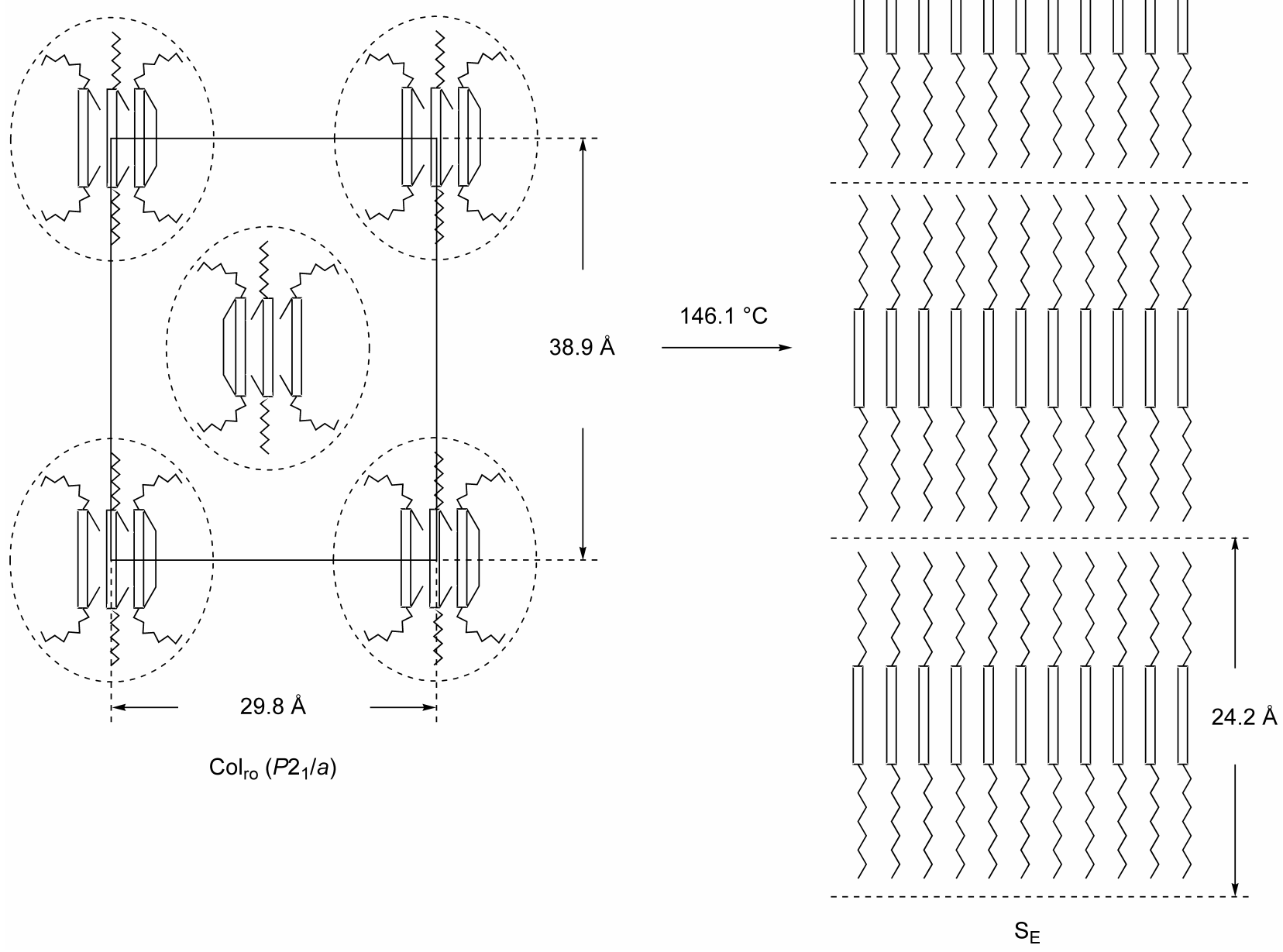

Figure S-19. Proposed phase structure models for the $\mathrm{Col}_{\mathrm{ro}}$ and $\mathrm{S}_{\mathrm{E}}$ mesophases of compound $\mathbf{2 b}$. 